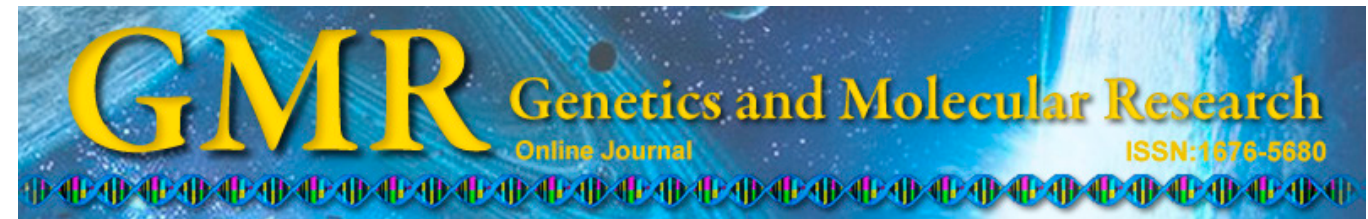

\title{
COL1A1 gene -1997G/T polymorphism and risk of osteoporosis in postmenopausal women: a meta-analysis
}

\author{
K.H. Yu ${ }^{1,2}$, J. Tang ${ }^{1}$, C.Q. Dai ${ }^{1}$, Y. Yu ${ }^{1}$ and J.J. Hong ${ }^{1}$ \\ ${ }^{1}$ Department of Orthopedic Surgery, \\ The Second Affiliated Hospital of Wenzhou Medical University, Wenzhou, \\ China \\ ${ }^{2}$ Department of Traumatic Orthopedics, \\ Shandong Provincial Hospital Affiliated to Shandong University, Jinan, \\ Shandong, China \\ Corresponding author: J.J. Hong \\ E-mail: hongmed@126.com
}

Genet. Mol. Res. 14 (3): 10991-10998 (2015)

Received February 10, 2015

Accepted June 15, 2015

Published September 21, 2015

DOI http://dx.doi.org/10.4238/2015.September.21.11

\begin{abstract}
Studies investigating the association between the COL1A1 gene $-1997 \mathrm{G} / \mathrm{T}$ polymorphism and the risk of osteoporosis in postmenopausal women have reported conflicting results. We performed a meta-analysis based on the evidence currently available from the literature to make a more precise estimation of this relationship. We conducted searches of the published literature in the PubMed and Embase databases up to September 2014. We estimated the pooled odds ratios with their $95 \%$ confidence intervals to assess the associations using fixed- or random-effect models. Publication bias was investigated by Begg's funnel plot. Meta-analysis was performed using the STATA package version 12.0. No significant association was found between the $-1997 \mathrm{G} / \mathrm{T}$ polymorphism in the COL1A1 gene and osteoporosis risk in the total population analysis (TT vs $\mathrm{GG}$ : $\mathrm{OR}=1.28,95 \% \mathrm{CI}=0.76$ 2.17; $\mathrm{TT}$ vs $\mathrm{GT}: \mathrm{OR}=1.04,95 \% \mathrm{CI}=0.60-1.78$; dominant model: $\mathrm{OR}=$
\end{abstract}


$0.84,95 \% \mathrm{CI}=0.50-1.40$; recessive model: $\mathrm{OR}=1.18,95 \% \mathrm{CI}=0.84$ 1.66). In a subgroup analysis by nationality, the results also showed that no significant associations between the COL1A1 gene -1997G/T polymorphism and osteoporosis risk existed in either Caucasian or Asian populations. No evidence of publication bias was found. In conclusion, the COL1A1 gene $-1997 \mathrm{G} / \mathrm{T}$ polymorphism might not be a risk factor for osteoporosis in postmenopausal women. Further large and well-designed studies are needed to confirm these conclusions.

Key words: COL1A1; Gene polymorphism; Osteoporosis; Meta-analysis

\section{INTRODUCTION}

Osteoporosis is a systemic skeletal disease characterized by low bone mineral density (BMD) and microarchitectural deterioration of bone leading to increased bone fragility and a high risk of fracture. Statistically, 30\% of women and $12 \%$ of men might suffer from osteoporosis at some point during their lifetime; accordingly, osteoporosis is becoming a major economic burden on society and on families (Hong et al., 2007). Over the past few years, the following risk factors have been shown to be related to osteoporosis outcome: race, heredity and constitution (family history), gender (female $>$ male), age (postmenopausal women in particular), body build (slender, small, and thin individuals), calcium deficiency, alcohol, smoking, excessive intake of salt and phosphorus, insufficient exercise (long-term bedridden), decreases in exercise capacity, among others (Suzuki, 2001). In addition, the pathogenesis of osteoporosis is partly influenced by multiple genetic factors (Shin et al., 2013).

Collagen is the most abundant protein of the bone matrix, accounting for approximately $80 \%$ of the total proteins in bone tissue. The alteration of collagen properties and of its relative quantity in the bone matrix can affect the mechanical features of bone tissue and increase the susceptibility to fragility fracture.

Collagen is composed of two peptide chains, alpha 1 and alpha 2, that are present at a 2:1 ratio in collagen fiber, and are encoded by the COL1A1 and COL1A2 genes. COLIA1 has been mapped to the long arm of chromosome 17 (17q21.33). Over 400 human disease-associated mutations have been identified within the COL1A1 gene, the majority of which are related to osteoporosis (Masoodi et al., 2013). Previous studies on COL1A1 have largely focused on the relationship between osteoporosis and the COLA1 Sp1 binding site polymorphism, which has been regarded as a predictor of reduced BMD and an increased risk of fragility fractures (Mann et al., 2001; Ralston et al., 2006). In addition, the -1997G/T (rs1107946) polymorphism has been identified in the COL1A1 upstream regulatory region; $-1997 \mathrm{G} / \mathrm{T}$ is in linkage disequilibrium with the $\mathrm{Sp} 1$ polymorphism (Garcia-Giralt et al., 2002; Jin et al., 2009).

To date, several studies have investigated the relationship between the COL1A1 $-1997 \mathrm{G} / \mathrm{T}$ polymorphism and osteoporosis risk in postmenopausal women. However, the conclusions from these studies have been conflicting. Furthermore, no meta-analysis data on the correlation of the COL1A1 -1997G/T polymorphism with the susceptibility to osteoporosis in postmenopausal women are currently available. Therefore, to derive a more precise overall effect estimate, the present study aimed to evaluate the association between the COL1A1 $-1997 \mathrm{G} / \mathrm{T}$ polymorphism and the susceptibility to osteoporosis in postmenopausal women by performing a systematic review and meta-analysis of the literature. 


\section{MATERIAL AND METHODS}

\section{Selection of studies}

Publications were identified by a systematic electronic search of the PubMed and Embase databases with the following key words: "osteoporosis" or "CYP1A1" and "-1997G/T" or "rs1107946" or "genotype" or "polymorphism". The last search was updated in September 2014. We did not set any restriction on the language of the published literature. In addition, the reference lists of the included articles and relevant meta-analyses were manually searched to identify additional relevant studies. Studies reported by the same authors were checked for possible overlapping participant groups.

\section{Inclusion and exclusion criteria}

The inclusion criteria used for the article selection in this meta-analysis were as follows: 1) case-control studies; 2) studies assessing the association of the $-1997 \mathrm{G} / \mathrm{T}$ polymorphism in the COL1A1 gene with osteoporosis risk; 3) provision of sufficient information for estimating the OR with its $95 \% \mathrm{CI}$; and 4) provision of available data to acquire the genotype frequency of the COL1A1 gene -1997G/T polymorphism. Major exclusion criteria were: 1) no control population; 2) no available genotype frequency; and 3) duplicated studies.

\section{Data extraction}

Two investigators (K.H. Yu and J. Tang) independently reviewed and extracted the information from all included publications using a standardized protocol, according to the inclusion and exclusion criteria. In the case of disagreement, discrepancies were resolved by discussion. The following characteristics were collected from each study: name of first author, year of publication, region of the first or corresponding author, ethnicity, number of cases and controls, number of genotypes, and evidence of Hardy-Weinberg equilibrium (HWE); these characteristics are listed in Table 1.

\section{Statistical analysis}

We predicted the contribution of the COL1A1 gene $-1997 \mathrm{G} / \mathrm{T}$ polymorphism to the risk of osteoporosis utilizing the STATA package version 12.0 (Stata Corporation, College Station, TX, USA). The strength of association was estimated by calculating summary crude ORs and the corresponding 95\%CIs under a co-dominant model (TT vs GG, TT vs GT), a dominant model $(\mathrm{GG}+\mathrm{GT} v s \mathrm{TT})$, and a recessive model $(\mathrm{TT}+\mathrm{GT}$ vs GG). The Fisher exact test was used to assess HWE with the significance set at $\mathrm{P}<0.05$. Between-study heterogeneities were estimated using the $\mathrm{I}^{2}$ test. $\mathrm{I}^{2}$ represents the variability that can be attributed to heterogeneity rather than chance. $I^{2}$ values of 25,50 , and $75 \%$ were defined as low, moderate, and high estimates, respectively. When a significant $\mathrm{I}^{2}>50 \%$ indicated heterogeneity across studies, the random-effect model was used for meta-analysis; else, the fixed-effect model was used. To evaluate ethnicity-specific effects, subgroup analyses were performed to explore and explain the diversity among the results of the different studies. Sensitivity analysis was performed through random-effect model values compared to the fixed effects. Publication bias was investigated by funnel plot and Begg's funnel plot. 


\section{RESULTS}

\section{Characteristics of the studies included}

Based on the search criteria, 24 articles were identified (Figure 1). Of these, 16 papers were excluded after review of the title or abstract because of obvious irrelevance to our study aim. In addition, one duplicated publication and two meta-analyses were excluded. An additional paper did not have a control group and was further excluded. Therefore, only four studies of the association between the COL1A1 gene -1997G/T polymorphism and osteoporosis risk were selected for this meta-analysis (Selezneva et al., 2008; Husted et al., 2009; Li et al., 2010; Singh et al., 2013). Two studies were conducted in Europe and two in Asia. The publishing years of the studies included ranged from 2008 to 2013. The source of controls was primarily based on healthy populations. The HWE test was performed on the genotype distribution of the controls, all of which were in HWE $(\mathrm{P}>0.05)$. The baseline characteristics and methodological quality of all studies included are summarized in Table 1.

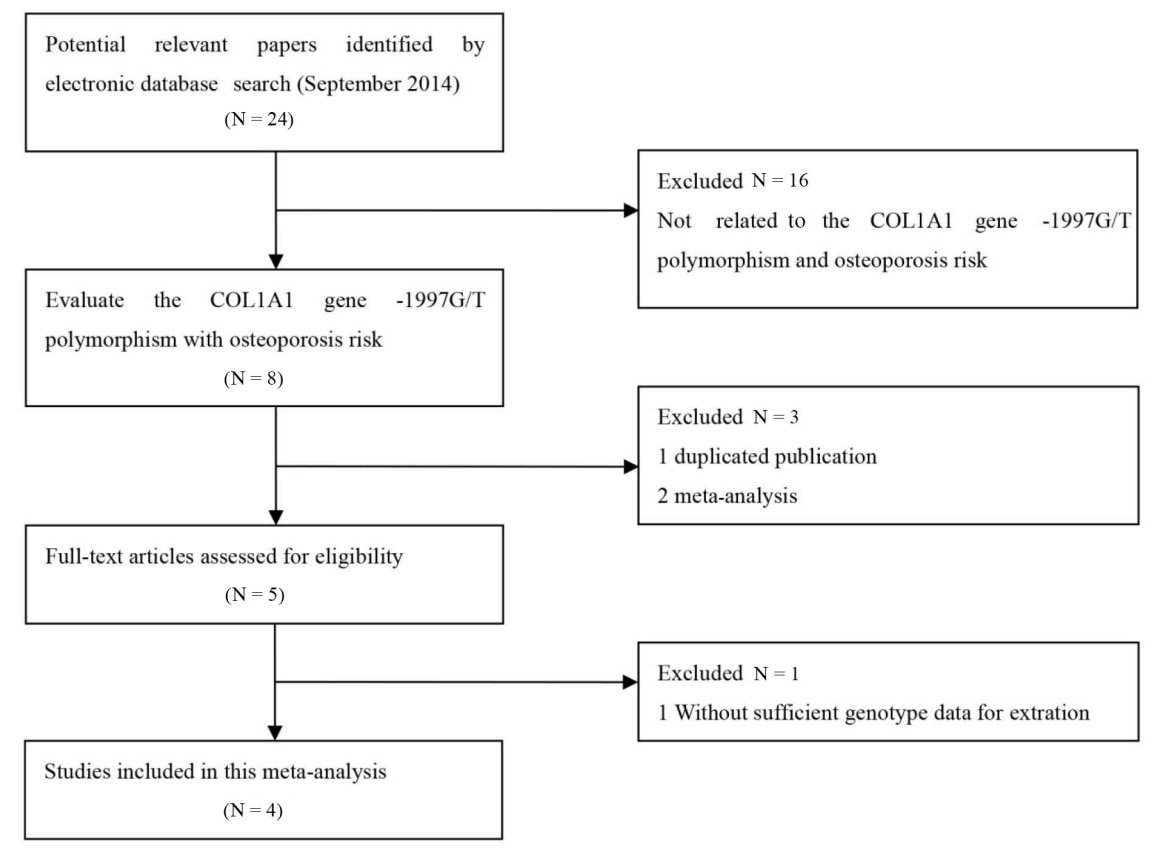

Figure 1. Flow chart showing study selection procedure.

Table 1. Characteristics of the studies included for meta-analysis.

\begin{tabular}{|c|c|c|c|c|c|c|c|c|c|c|c|}
\hline \multirow[t]{2}{*}{ Study included } & \multirow[t]{2}{*}{ Year } & \multirow[t]{2}{*}{ Area } & \multirow[t]{2}{*}{ Race } & \multirow[t]{2}{*}{ Cases/Controls } & \multicolumn{3}{|c|}{ Genotypes for cases } & \multicolumn{3}{|c|}{ Genotypes for controls } & \multirow[t]{2}{*}{ HWE test } \\
\hline & & & & & GG & GT & TT & GG & GT & TT & \\
\hline Selezneva et al. & 2008 & Russia & Caucasians & $124 / 150$ & 84 & 36 & 4 & 90 & 55 & 5 & 0.33 \\
\hline Husted et al. & 2009 & Denmark & Caucasians & $228 / 226$ & 158 & 63 & 7 & 175 & 46 & 5 & 0.35 \\
\hline Li et al. & 2010 & China & Asians & $212 / 106$ & 82 & 99 & 31 & 51 & 45 & 10 & 0.99 \\
\hline Singh et al. & 2013 & India & Asians & $145 / 117$ & 99 & 43 & 3 & 83 & 28 & 6 & 0.09 \\
\hline
\end{tabular}




\section{Quantitative synthesis}

A summary of the meta-analysis findings of the association between the COL1A1 gene -1997G/T polymorphism and osteoporosis risk is shown in Figure 2 and Table 2. Metaanalysis results showed that the COL1A1 $-1997 \mathrm{G} / \mathrm{T}$ polymorphism might not be associated with osteoporosis risk (TT $v s$ GG: $\mathrm{OR}=1.28,95 \% \mathrm{CI}=0.76-2.17$; TT $v s$ GT: $\mathrm{OR}=1.04$, $95 \% \mathrm{CI}=0.60-1.78$; dominant model: $\mathrm{OR}=0.84,95 \% \mathrm{CI}=0.50-1.40$; recessive model: $\mathrm{OR}=$ $1.18,95 \% \mathrm{CI}=0.84-1.66)$. Sensitivity analyses were conducted by altering the statistic models (TT vs GG: $\mathrm{OR}=1.21,95 \% \mathrm{CI}=0.64-2.30$; $\mathrm{TT}$ vs GT: $\mathrm{OR}=1.04,95 \% \mathrm{CI}=0.60-1.82$; dominant model: $\mathrm{OR}=0.86,95 \% \mathrm{CI}=0.49-1.50$; recessive model: $\mathrm{OR}=1.20,95 \% \mathrm{CI}=0.94-1.51$ ). No material alteration was detected, indicating that our results were statistically robust.

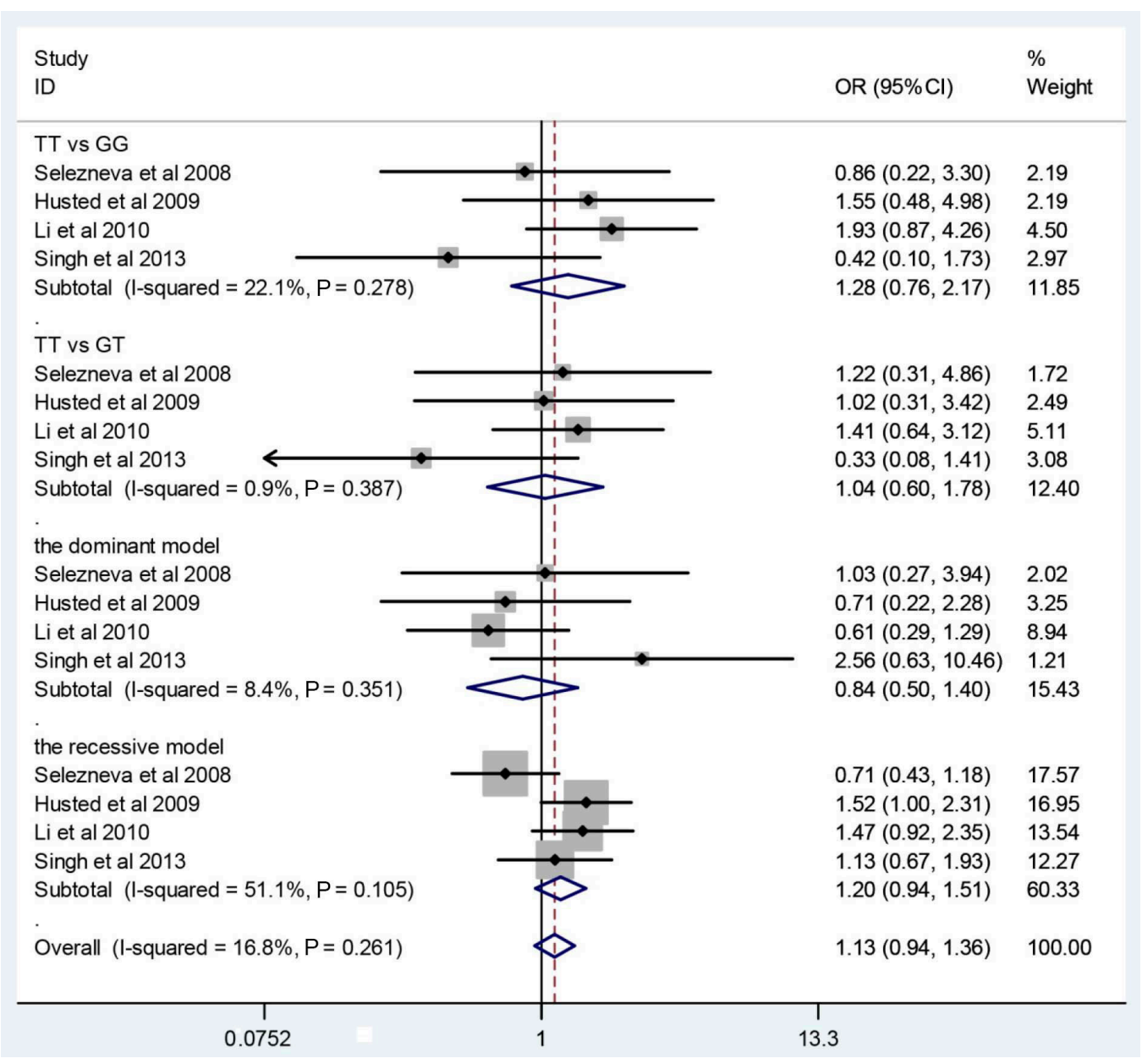

Figure 2. Forest plot of osteoporosis risk in postmenopausal women associated with the COL1A1 gene -1997G/ $\mathrm{T}$ polymorphism. The squares and horizontal lines correspond to the study-specific odds ratios (OR) and $95 \%$ confidence intervals (CI). 
Table 2. Summary ORs and $95 \% \mathrm{CI}$ of the COL1A1 gene $-1997 \mathrm{G} / \mathrm{T}$ polymorphism with osteoporosis risk.

\begin{tabular}{|c|c|c|c|c|c|c|c|c|c|c|}
\hline \multirow{3}{*}{$\begin{array}{l}\text { Subgroup } \\
\text { Overall }\end{array}$} & \multirow{3}{*}{$\begin{array}{l}\text { Genetic model } \\
\text { TT } v_{s} \mathrm{GG}\end{array}$} & \multirow{2}{*}{\multicolumn{2}{|c|}{$\frac{\text { Sample size }}{\text { Case/Control }}$}} & \multirow{3}{*}{$\begin{array}{l}\text { Type of model } \\
\text { Fixed }\end{array}$} & \multicolumn{2}{|c|}{ Test of heterogeneity } & \multicolumn{2}{|c|}{ Test of association } & \multicolumn{2}{|c|}{ Test of publication bias } \\
\hline & & & & & \multirow{2}{*}{$\begin{array}{c}\mathrm{I}^{2} \\
22.1 \%\end{array}$} & \multirow{2}{*}{$\frac{P}{0.28}$} & \multirow{2}{*}{$\begin{array}{c}\mathrm{OR} \\
1.28\end{array}$} & \multirow{2}{*}{$\begin{array}{c}95 \% \mathrm{CI} \\
0.76-2.17\end{array}$} & \multirow{2}{*}{$\begin{array}{c}\mathrm{z} \\
0.34\end{array}$} & \multirow{2}{*}{$\begin{array}{c}P \\
0.73\end{array}$} \\
\hline & & 709 & 599 & & & & & & & \\
\hline & TT $v s \mathrm{GT}$ & & & Fixed & $0.9 \%$ & 0.39 & 1.04 & $0.60-1.78$ & 0.34 & 0.73 \\
\hline & Dominant model & & & Fixed & $8.4 \%$ & 0.35 & 0.84 & $0.50-1.40$ & 0.34 & 0.73 \\
\hline & Recessive model & & & Random & $51.1 \%$ & 0.11 & 1.18 & $0.84-1.66$ & 0.34 & 0.73 \\
\hline \multirow[t]{4}{*}{ Caucasians } & TT $v s \mathrm{GG}$ & 352 & 376 & Fixed & $0.0 \%$ & 0.52 & 1.20 & $0.50-2.88$ & 0.00 & 1.00 \\
\hline & $\mathrm{TT} v s \mathrm{GT}$ & & & Fixed & $0.0 \%$ & 0.85 & 1.10 & $0.44-2.75$ & 0.00 & 1.00 \\
\hline & Dominant model & & & Fixed & $0.0 \%$ & 0.68 & 0.84 & $0.35-2.00$ & 0.00 & 1.00 \\
\hline & Recessive model & & & Random & $80.6 \%$ & 0.02 & 1.06 & $0.50-2.21$ & 0.00 & 1.00 \\
\hline \multirow[t]{4}{*}{ Asians } & TT $v s \mathrm{GG}$ & 357 & 223 & Random & $70.5 \%$ & 0.07 & 1.01 & $0.23-4.43$ & 0.00 & 1.00 \\
\hline & $\mathrm{TT} v_{s} \mathrm{GT}$ & & & Random & $66.3 \%$ & 0.09 & 0.78 & $0.19-3.18$ & 0.00 & 1.00 \\
\hline & Dominant model & & & Random & $67.8 \%$ & 0.08 & 1.10 & $0.28-4.39$ & 0.00 & 1.00 \\
\hline & Recessive model & & & Fixed & $0.0 \%$ & 0.47 & 1.31 & $0.92-1.86$ & 0.00 & 1.00 \\
\hline
\end{tabular}

OR, odds ratio; CI, confidence interval.

In subgroup analysis by ethnicity, the studies included were divided into Caucasian and Asian populations; no significant association was found between the COL1A1 gene $-1997 \mathrm{G} / \mathrm{T}$ polymorphism and osteoporosis risk in Caucasians (TT vs GG: OR $=1.20,95 \% \mathrm{CI}$ $=0.50-2.88$; TT $v_{S} \mathrm{GT}: \mathrm{OR}=1.10,95 \% \mathrm{CI}=0.44-2.75 ;$ dominant model: $\mathrm{OR}=0.84,95 \% \mathrm{CI}=$ 0.35-2.00; recessive model: $\mathrm{OR}=1.06,95 \% \mathrm{CI}=0.50-2.21)$ or Asians (TT $v s \mathrm{GG}: \mathrm{OR}=1.01$, $95 \% \mathrm{CI}=0.23-4.43$; $\mathrm{TT} v \mathrm{GT}: \mathrm{OR}=0.78,95 \% \mathrm{CI}=0.19-3.18$; dominant model: $\mathrm{OR}=1.10$, $95 \% \mathrm{CI}=0.28-4.39$; recessive model: $\mathrm{OR}=1.31,95 \% \mathrm{CI}=0.92-1.86$ ).

\section{Publication bias}

Potential publication bias of the literature was assessed by Begg's funnel plot. The Begg's funnel plot was used to measure the asymmetry of the funnel plot. The results of the Begg funnel plot test are shown in Table 2 and Figure 3. Results showed that there was no publication bias (all $\mathrm{P}>0.05$ ).

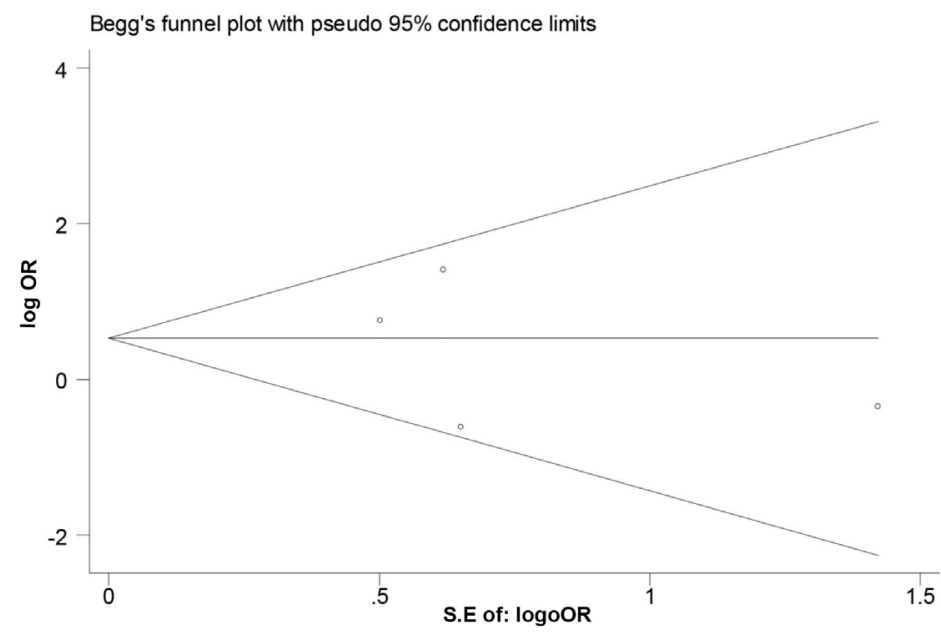

Figure 3. Funnel plot of the COL1A1 gene-1997G/T polymorphism and susceptibility of osteoporosis for TT vs GG. 


\section{DISCUSSION}

Osteoporosis is a one of the typical diseases that develops from a long-standing lifestyle and is induced by multiple factors. With the growing aging population, the number of patients with osteoporosis is expected to increase to 2.6 million by 2025 and to 4.5 million by 2050 (Abrahamsen et al., 2009). The pathogenesis of osteoporosis is complex and multifactorial and is still not fully understood. In recent years, accumulating evidence has indicated that osteoporosis is determined by complex interactions between environmental and genetic factors. One of the most important candidate genes for the predisposition to osteoporosis is the COL1A1 gene, which encodes the alpha 1 protein chain of collagen, the major protein of bone. Previous research has shown that the COL1A1 gene Sp1 polymorphism is a reliable predictor of osteoporosis risk (Mann et al., 2001). To date, a number of studies have focused on the association between the COL1A1 gene $-1997 \mathrm{G} / \mathrm{T}$ polymorphism and the risk of osteoporosis in postmenopausal women. However, the observed associations of these studies were inconsistent. To help clarify the earlier inconclusive findings with those from several recently published studies, we conducted this meta-analysis. The aim of meta-analysis is to combine similar kinds of studies to increase the effective sample size and statistical power, and thereby obtain a more authentic and reliable result.

This is the first systematic study of the association between the COL1A1 gene -1997G/ $\mathrm{T}$ polymorphism and osteoporosis risk in postmenopausal women using meta-analysis. Our study quantitatively assessed the association between the $-1997 \mathrm{G} / \mathrm{T}$ polymorphism and the susceptibility to osteoporosis. Ultimately, four case-control studies were included and assessed in this meta-analysis, involving a total of 709 patients with osteoporosis and 599 healthy controls. The results of the meta-analysis revealed that no significant association could be identified between the COL1A1 gene -1997G/T polymorphism and the risk of osteoporosis in postmenopausal women. Considering that the result might be affected by ethnicity, we performed a race-related subgroup analysis; however, no significant association was found in either Caucasian or Asian populations. No evidence was found to suggest the existence of publication bias in this meta-analysis. However, as the eligible study number was limited in the meta-analysis, caution should be exercised when considering this conclusion.

The potential function of the $-1997 \mathrm{G} / \mathrm{T}$ polymorphism might be affected through gene-gene interactions. The linkage disequilibrium (-1997G/T, 21663IndelT, and Sp1 polymorphism) in the COL1A1 gene might synergistically regulate COL1A1 transcription by affecting DNA-protein interactions and thereby increase the risk of osteoporosis (Jin et al., 2009). In addition, the COL1A1 and VDR genes might also synergistically increase the susceptibility to osteoporosis (Uitterlinden et al., 2001). Furthermore, gene-environment interactions should also be taken into consideration in future analysis.

In this meta-analysis, no significant between-study heterogeneities were identified in the heterogeneity tests, indicating that our results were unbiased, and no obvious publication bias was shown to exist. However, some limitations of this study should be acknowledged. First, our meta-analysis was based on unadjusted OR estimates because not all published studies presented adjusted ORs, or when they did, the ORs were not adjusted by the same potential confounders, such as age, gender, and exposures. Lacking information for data analysis might cause serious confounding bias. Second, the number of studies and the number of subjects in the studies included in the meta-analysis by specific subgroups were small. Additionally, meta-analysis is retrospective research that is subject to methodological limitations. 
In conclusion, our meta-analysis suggests that no association between the COL1A1 gene -1997G/T polymorphism and osteoporosis risk exists in postmenopausal women. As few studies are available in this field and current evidence remains limited, it should be emphasized that it will be necessary to conduct large-scale studies with adequate methodological quality in order to come to a definitive conclusion on this issue.

\section{Conflicts of interest}

The authors declare no conflict of interest.

\section{ACKNOWLEDGMENTS}

Research supported by the Administration of Traditional Chinese Medicine of Zhejiang Province (\#2010ZB099,\#2013ZQ024) and the Science and Technology Planning Project of Wenzhou (\#Y20130183).

\section{REFERENCES}

Abrahamsen B, van Staa T, Ariely R, Olson M, et al. (2009). Excess mortality following hip fracture: a systematic epidemiological review. Osteoporos. Int. 20: 1633-1650.

Garcia-Giralt N, Nogués X, Enjuanes A, Puig J, et al. (2002). Two new single-nucleotide polymorphisms in the COL1A1 upstream regulatory region and their relationship to bone mineral density. J. Bone Miner. Res. 17: 384-393.

Hong X, Hsu YH, Terwedow H, Tang G, et al. (2007). Association of the methylenetetrahydrofolate reductase C677T polymorphism and fracture risk in Chinese postmenopausal women. Bone 40: 737-742.

Husted LB, Harsløf T, Gonzalez-Bofill N, Schmitz A, et al. (2009). Haplotypes of promoter and intron 1 polymorphisms in the COLIA1 gene are associated with increased risk of osteoporosis. Calcif. Tissue Int. 84: 85-96.

Jin H, van't Hof RJ, Albagha OM and Ralston SH (2009). Promoter and intron 1 polymorphisms of COL1A1 interact to regulate transcription and susceptibility to osteoporosis. Hum. Mol. Genet. 18: 2729-2738.

Li Y, Shen B, Huang Q, Cao F, et al. (2010). Study on the correlation between the polymorphism of $-1997 \mathrm{~g} / \mathrm{T}$ site of collagen I alpha 1 gene and bone mineral density in postmenopausal women. Sichuan Da Xие Хие Baо Yi Xие Ban 41: 121-124.

Mann V, Hobson EE, Li B, Stewart TL, et al. (2001). A COL1A1 Sp1 binding site polymorphism predisposes to osteoporotic fracture by affecting bone density and quality. J. Clin. Invest. 107: 899-907.

Masoodi TA, Alsaif MA, Al Shammari SA and Alhamdan AA (2013). Evaluation and identification of damaged single nucleotide polymorphisms in COL1A1 gene involved in osteoporosis. Arch. Med. Sci. 9: 899-905.

Ralston SH, Uitterlinden AG, Brandi ML, Balcells S, et al. (2006). Large-scale evidence for the effect of the COLIA1 Sp1 polymorphism on osteoporosis outcomes: the GENOMOS study. PLoS Med. 3: e90.

Selezneva LI, Khusainova RI, Nurlygaianov RZ, Fazlyeva EA, et al. (2008). Association of polymorphisms and haplotypes in the 5' region of COLIA1 gene with the risk of osteoporotic fractures in Russian women from Volga-Ural region. Genetika 44: 219-225.

Shin MH, Choi JS, Rhee JA, Lee YH, et al. (2013). Association between methylenetetrahydrofolate reductase C677T polymorphism and bone mineral density: the Dong-gu Study and the Namwon Study. J. Korean Med. Sci. 28: 965968.

Singh M, Singh P, Singh S, Juneja PK, et al. (2013). A haplotype derived from the common variants at the $-1997 \mathrm{G} / \mathrm{T}$ and Sp1 binding site of the COL1A1 gene influences risk of postmenopausal osteoporosis in India. Rheumatol. Int. 33: 501-506.

Suzuki T (2001). Risk factors for osteoporosis in Asia. J. Bone Miner. Metab. 19: 133-141.

Uitterlinden AG, Weel AE, Burger H, Fang Y, et al. (2001). Interaction between the vitamin D receptor gene and collagen type I alpha1 gene in susceptibility for fracture. J. Bone Miner. Res. 16: 379-385. 